\title{
Observation of Nociceptive Processing: Effect of Intra-Epidermal Electric Stimulus Properties on Detection Probability and Evoked Potentials
}

\author{
Boudewijn van den Berg ${ }^{1}$ (D) Jan R. Buitenweg ${ }^{1}$ (D)
}

Received: 15 June 2020 / Accepted: 25 December 2020 / Published online: 18 January 2021

(c) The Author(s) 2021

\begin{abstract}
Monitoring nociceptive processing is a current challenge due to a lack of objective measures. Recently, we developed a method for simultaneous tracking of psychophysical detection probability and brain evoked potentials in response to intraepidermal stimulation. An exploratory investigation showed that we could quantify nociceptive system behavior by estimating the effect of stimulus properties on the evoked potential (EP). The goal in this work was to accurately measure nociceptive system behavior using this method in a large group of healthy subjects to identify the locations and latencies of EP components and the effect of single- and double-pulse stimuli with an inter-pulse interval of 10 or $40 \mathrm{~ms}$ on these EP components and detection probability. First, we observed the effect of filter settings and channel selection on the EP. Subsequently, we compared statistical models to assess correlation of EP and detection probability with stimulus properties, and quantified the effect of stimulus properties on both outcome measures through linear mixed regression. We observed lateral and central EP components in response to intra-epidermal stimulation. Detection probability and central EP components were positively correlated to the amplitude of each pulse, regardless of the inter-pulse interval, and negatively correlated to the trial number. Both central and lateral EP components also showed strong correlation with detection. These results show that both the observed EP and the detection probability reflect the various steps of processing of a nociceptive stimulus, including peripheral nerve fiber recruitment, central synaptic summation, and habituation to a repeated stimulus.
\end{abstract}

Keywords Electroencephalography $\cdot$ Psychophysics $\cdot$ Evoked potential $\cdot$ Detection threshold $\cdot$ Detection probability $\cdot$ Intraepidermal electric stimulation · Nociception · Nociceptive Processing · Peripheral nerve fiber recruitment · Central synaptic summation $\cdot$ Habituation

\section{Introduction}

A major challenge in the development of pain biomarkers is the complex nature of the pain experience, as it is determined by a significant amount of supra-spinal processing of the initial sensory input (Apkarian et al. 2005). Developing methods to accurately measure the relation between a welldefined sensory input, brain activation, and pain perception might 1 day lead to more objective mechanism-based pain

Handling Editor: Geertjan Huiskamp.

Boudewijn van den Berg

b.van.den.berg@utwente.nl

1 Biomedical Signals and Systems, Technical Medical Centre, University of Twente, PO Box 217, 7500 AE Enschede,

The Netherlands biomarkers. A well-defined nociceptive sensory input can be generated by preferential stimulation of nociceptive $A \delta$ afferents in the skin using intra-epidermal electric stimulation (Inui et al. 2002). This technique has been shown to preferentially activate nociceptive afferents when applied at less than two times the detection threshold (Mouraux et al. 2010; Poulsen et al. 2020). Recently, we developed a method to concentrate stimulation around this (drifting) detection threshold, and measure stimulus-response pairs and evoked potentials in response to these nociceptive stimuli (van den Berg et al. 2020).

Based on acquired stimulus-response pairs, a psychometric function for the detection probability can be determined which is characterized by a detection threshold and a slope. Recent research has demonstrated that the detection threshold can be used to observe both short-term and long-term effects of experimental pain conditioning. Conditioned pain 
modulation by immersion of one foot in ice water resulted in a direct increase of the detection threshold of single-pulse intra-epidermal stimuli (Doll et al. 2014). On the other hand, 1-h application of an $8 \%$ capsaicin patch resulted in a longterm increase of the detection threshold to intra-epidermal stimuli (Doll et al. 2016a, b). More specifically, detection thresholds to single-pulse intra-epidermal stimuli were significantly increased on days 2 to 7 following capsaicin application, while detection thresholds to double-pulse intraepidermal stimuli were significantly increased on days 7 to 28 after capsaicin application. The difference between both stimulus types was that by using two or more pulses, we also observed the effect of temporal summation on nociceptive processing, which lead to a significant decrease of the detection threshold and increase of the slope in the case of double-pulse intra-epidermal stimuli (Doll et al. 2016a, b).

Centering stimulus amplitudes around the detection threshold allows for the measurement of evoked potentials in response to nociceptive intra-epidermal stimulation. Earlier studies showed that intra-epidermal stimulation at twice the detection threshold results in an evoked potential waveform that is sensitive to experimental pain conditioning such as the intra-epidermal injection of capsaicin (Liang et al. 2016) and high-frequency stimulation (Manresa et al. 2018). Typically this evoked potential included an early contralateral negative peak around $150 \mathrm{~ms}$ referred to as the $\mathrm{N} 1$ (Mouraux 2014), a central negative peak observed between 130 and $150 \mathrm{~ms}$ (Liang et al. 2016) or 220-230 ms (Mouraux et al. 2014), and a central positive peak between 290 and $330 \mathrm{~ms}$ (Liang et al. 2016) or 360-370 ms (Mouraux et al. 2014). This difference in latencies might be partly explained by the difference in filter settings used in both studies. Nevertheless, a systematic evaluation of the influence of filter settings on intra-epidermal evoked potential waveforms and topographies has not been done so far. Intra-epidermal evoked potential waveforms were also shown to be affected by the number of pulses, as the N1, N2 and P2 were shown to be larger and more reliable when multiple electric current pulses were applied, while the evoked potential latencies and response times remained the same (Mouraux et al. 2014). However, an evaluation of the influence of the interval between those pulses on the resulting evoked potential was not done yet.

After a technical pilot study (van den Berg et al. 2020), we designed a new study with the combined measurement of detection thresholds and evoked potentials to accurately quantify the effect of intra-epidermal stimulus properties, i.e. number of pulses and inter-pulse interval, on both outcome measures in healthy individuals. More specifically, we wanted to (1) confirm the presence of previously observed EP components in response to intra-epidermal electric stimulation, (2) determine at which scalp locations and which latencies the observed EP components are maximal and
(3) analyze in detail how these components and detection thresholds are influenced by intra-epidermal stimulus properties in healthy subjects, using (generalized) linear mixed regression models. After exploring the effect of various filter settings on the EP waveform, we investigated which model most effectively captures the data and studied the effect of detection, pulse amplitudes, trial number and the interaction between detection and trial number. In this way, we aimed to obtain new insights and directions for the design of future studies employing this method to study alterations in nociceptive function.

\section{Methods}

The experiments presented in this work include measurements of the detection threshold and the EEG with respect to intra-epidermal stimuli on a single occasion at the University of Twente, the Netherlands. All experiments were approved by the local Medical Review and Ethics Committee and in accordance with the declaration of Helsinki.

\section{Participants}

A total of 30 healthy participants (20 males and 10 females, age $23.0 \pm 3.4$, 4 left-handed) were included in this study. To be included, participants had to have an age between 18 and 40 years old. Exclusion criteria were skin abnormalities at the site of stimulation, diabetes, implanted stimulation devices, pregnancy, usage of analgesics within $24 \mathrm{~h}$ before the experiment, the consumption of alcohol or drugs within $24 \mathrm{~h}$ before the experiments, pain complaints at the time of the experiment, a medical history of chronic pain or any language problems that would impede communication with the participant. All participants provided written informed consent and received a monetary compensation of $€ 20$ for participation in the experiment.

\section{Stimuli}

Stimuli consisted of square-wave electrical current pulses generated by a constant current stimulator (NociTRACK AmbuStim, University of Twente, Enschede, The Netherlands) and were applied intra-epidermal to achieve preferential activation of A $\delta$-fibers (Inui et al. 2002; Mouraux et al. 2010). Stimulation was applied using a custom made electrode consisting of 5 inter-connected microneedles (Fig. 1). A previous validation study of this electrode showed that stimulation resulted in a sharp pricking sensation (Steenbergen 2012).

A previous study measuring the detection probability and detection thresholds in response to intra-epidermal stimulation observed a larger detection probability than would 
Fig. 1 Electrode for intra-epidermal stimulation, consisting of an array of 5 inter-connected microneedles embedded in a flexible layer of silicone

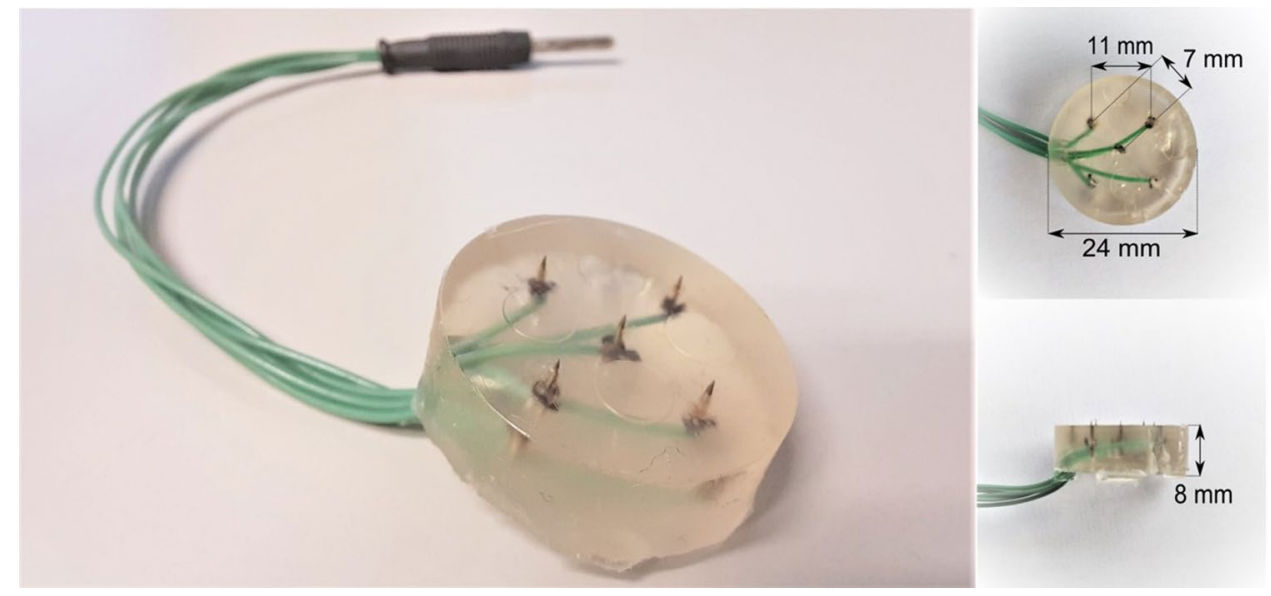

be expected based on probability summation for doublepulse stimuli with inter-pulse intervals on a range of 10 to $100 \mathrm{~ms}$, which suggests that such stimuli are amplified by a facilitatory mechanism (Doll et al. 2016a, b). In this study, we aimed to reproduce this increased detection probability of double-pulse stimuli, and to observe if this is related to an increase of evoked potential. As we wanted to remain well below the inter-pulse interval at which each pulse is observed individually of $200 \mathrm{~ms}$ (Lee et al. 2009) and well above the time required for nerve repolarization we used inter-pulse intervals of 10 and $40 \mathrm{~ms}$. As such, a total of three different settings was used:

- A single $210 \mu$ s pulse.

- A double $210 \mu$ s pulse with an inter-pulse interval of $10 \mathrm{~ms}$.

- A double $210 \mu$ s pulse with an inter-pulse interval of $40 \mathrm{~ms}$.

\section{Procedure}

Participants were seated in a comfortable chair and instructed to focus on the electrode. First, their initial detection threshold was approximated by a standard staircase procedure without inversion with a step size of $0.025 \mathrm{~mA}$. Subsequently, nociceptive detection thresholds were tracked simultaneously for the 3 stimulus types on 30 participants, with a total of 150 stimuli per stimulus type per participant. Participants were instructed to press and hold a button, and shortly release the button as soon as they felt a sensation that they ascribe to the application of a stimulus. While the button was pressed, the stimulator applied stimuli to the participant. Stimulus amplitudes were chosen according to an adaptive staircase procedure designed to converge towards and track a time-dependent psychophysical threshold (Doll et al. 2015). A set of five equidistant amplitudes with a step size of $0.025 \mathrm{~mA}$ was defined around the initial detection threshold, from which the next stimulus was randomly selected. A stimulus was identified as detected if the participant released the button within one second after the stimulus, and otherwise considered non-detected. This reaction time was measured internally by the stimulator as the time between stimulus onset and button release, with a resolution 35 microseconds. The set of equidistant amplitudes was decreased by $0.025 \mathrm{~mA}$ if a stimulus was detected and increased by $0.025 \mathrm{~mA}$ if a stimulus was nondetected. Subsequently, the next stimulus was selected from the updated set of equidistant amplitudes and applied after a uniformly randomized interval of 4.3 to $5.3 \mathrm{~s}$. This procedure was repeated until the end of the experiment (Fig. 2).

\section{Electroencephalography}

The scalp EEG was continuously recorded with a sampling rate of $1024 \mathrm{~Hz}$ using a REFA amplifier (TMSi B.V., Oldenzaal, the Netherlands) at $128 \mathrm{Ag} / \mathrm{AgCl}$ electrodes with a common average reference. Electrodes were placed on the scalp according to the international 10/5 system (Oostenveld and Praamstra 2001) and additional leads were placed on the earlobes. The participants were asked fix their gaze at a spot on the wall and blink as few times as possible while they pressed the response button and hence received stimuli. Participants with excessive electrode impedance ( $>5$ channels with $>20 \mathrm{k} \Omega$ ) were excluded. Therefore, a total of 25 participants (16 males and 9 females, age $23 \pm 3.6$, 1 lefthanded) were used for analysis.

EEG data was pre-processed using FieldTrip (Oostenveld et al. 2011), a Matlab toolbox for EEG and MEG signal processing. Contamination of the EEG by eye-blinks was corrected using an independent component analysis algorithm (Delorme et al. 2007). Subsequently, epochs with excessive EMG activity or movement artefacts were removed by visual inspection. The first 15 epochs were removed as no reliable estimate of the detection threshold was available for those trials. Furthermore, epochs in which the stimulus amplitude 


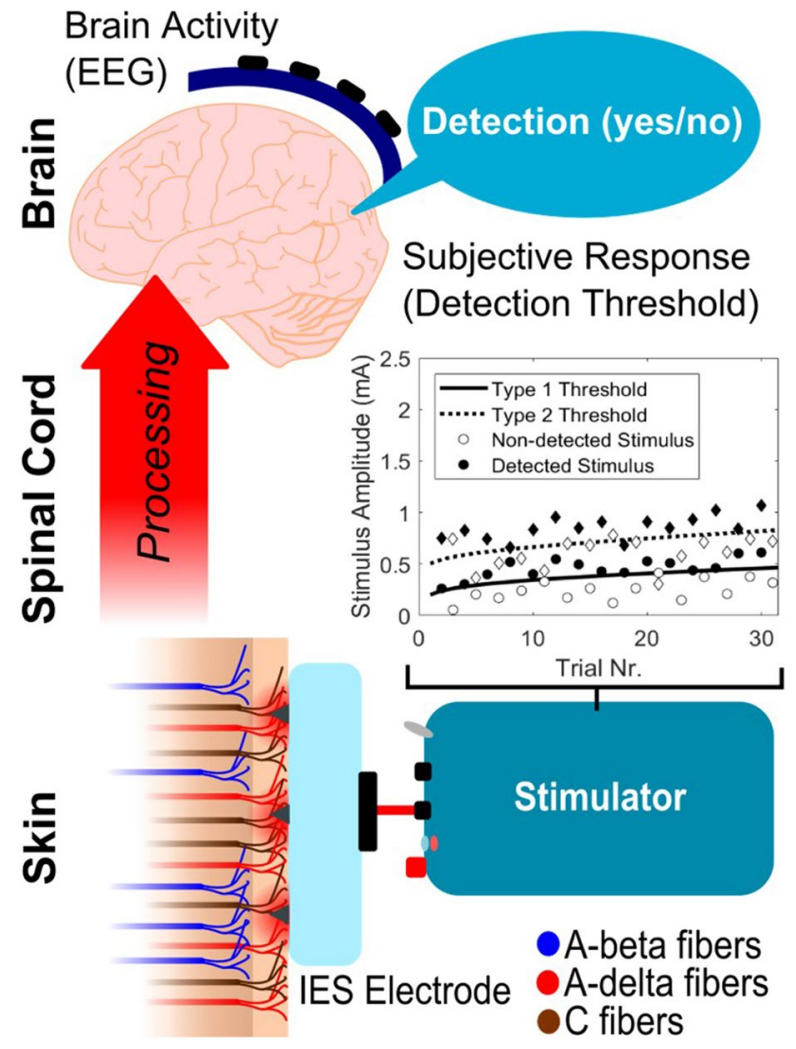

Fig. 2 We attempt to characterize nociceptive processing by simultaneously measuring the effect of intra-epidermal stimulus properties on psychophysical detection probability and brain evoked potentials. Multiple stimulus types (i.e. with a different number of pulses or inter-pulse interval) are generated with a varying amplitude close to the detection threshold. Stimuli preferentially activate nociceptive nerve fibers by using a specially designed needle electrode which protrudes into the epidermis of the skin using microneedles. Subject responses (detected or non-detected) and EEG corresponding to the applied stimuli are acquired and analyzed using (generalized) linear mixed regression to quantify the effect of stimulus properties on detection probability and evoked potentials

exceeded two times the tracked individual detection threshold were excluded from analysis. Based on earlier observations that opercular sources are first activated contralateral with respect to stimulation and lateralized afterwards (Garcia-Larrea et al. 2003), the channel topography for lefthanded subjects was inverted such that uneven numbers correspond to the contralateral side and even numbers to the ipsilateral side with respect to stimulation.

Epochs for EP analysis were extracted from the EEG using a window ranging from $0.5 \mathrm{~s}$ before until $1.0 \mathrm{~s}$ after the stimulus. To compare EP waveforms in the current study, with those previously observed in literature in response to intra-epidermal stimulation, grand average waveforms and topographies at $\mathrm{Cz}$ (average reference) and $\mathrm{T} 7-\mathrm{Fz}$, bandpass filtered at 0.1 to $40 \mathrm{~Hz}, 0.5$ to $45 \mathrm{~Hz}$ and 0.1 to $30 \mathrm{~Hz}$ and baseline corrected, were set side by side. Further analyses were performed using the waveforms bandpass filtered at 0.1 to $40 \mathrm{~Hz}$ to minimize signal loss and the potential bias induced by the high-pass filter (Acunzo et al. 2012).

Average latencies of three peaks in the EP were defined as follows. A first negative peak (N1), was defined as the most negative peak at T7-Fz between 130 and $170 \mathrm{~ms}$ after stimulus onset. A second negative peak (N2) was defined as the most negative peak at $\mathrm{Cz}$ between 170 and $300 \mathrm{~ms}$ after stimulus onset. Lastly, a positive peak (P2) was defined as the most positive peak at $\mathrm{Cz}$ between 300 and $500 \mathrm{~ms}$ after stimulus onset. To check if each of those peaks coincide with global peaks of EEG activity, peak latencies were compared with the butterfly plot and the global field power (Lehmann and Skrandies 1980).

To systematically study on which locations the N1, $\mathrm{N} 2$ and $\mathrm{P} 2$ are best observed, the SNR was computed for each channel, where SNR was defined as in (1), where $S(t)$ denotes the grand average potential at latency $t$ and $\sigma_{\text {baseline }}$ denotes the standard deviation of the grand average from -0.5 to $-0.3 \mathrm{~s}$ with respect to stimulus onset.

$S N R=\frac{|S(t)|}{\sigma_{\text {baseline }}}$

Subsequently, grand average EP waveforms were computed at derivations with a maximum SNR: T7-F4 at N1 and $\mathrm{N} 2$, and $\mathrm{CPz}-\mathrm{A} 1 \mathrm{~A} 2$ at $\mathrm{P} 2$. The effect of intra-epidermal stimulus properties on these EP waveforms was studied using linear mixed regression, which is further outlined in "Effect of Intra-Epidermal Stimulus Properties on Evoked Potential" section.

\section{Effect of Intra-Epidermal Stimulus Properties on Detection Probability}

Statistical analysis of stimulus-response pairs was performed in $\mathrm{R}$ with the lme4 toolbox (Bates et al. 2015). The effect of stimulus properties on the detection probability was estimated using logistic generalized linear mixed regression using a statistical model selected using the procedure outlined in "Model Selection" section. The track of individual and group level thresholds was estimated by performing generalized mixed regression over a moving window of 30 stimulus-response pairs. Subsequently, generalized mixed regression was performed over the entire dataset to establish accurate estimates of effect size and significance. The trial number was centered and scaled to speed up the model estimation process. Estimates of the threshold and slope were obtained using the estimated effect sizes, and corresponding standard errors were approximated using the Delta procedure (Faraggi et al. 2003; Moscatelli et al. 2012). Effect significance was assessed using type III Wald Chi-square statistics with a two-tailed test. As 26 tests are performed in this 
article, the significance level was set to $0.025 / 26 \approx 0.001$ after Bonferroni correction.

\section{Effect of Intra-Epidermal Stimulus Properties on Evoked Potential}

Statistical analysis of EEG data was performed in MATLAB 2017b (MathWorks, Inc.). The effect size of stimulus properties was evaluated using linear mixed regression (Van den Berg and Buitenweg 2018). Regression parameters were computed for every point in time at CPz-A1A2 and T7-F4 using a statistical model selected using the procedure oultined in "Model Selection" section. Model variables were centered and scaled to speed up the estimation progress. Subsequently, effect sizes and their corresponding $t$-values were estimated for every point in time by optimization of the restricted maximum likelihood. At component latencies (153, 213 and $418 \mathrm{~ms})$ significance of the effect sizes was assessed using the $t$-statistic with a two-tailed test using Satterthwaite's method for estimation of the degrees of freedom. Similar to the previous section, the significance level was set to 0.001 after Bonferroni correction.

\section{Model Selection}

We compared several models for statistical analysis of the detection probability and EEG, based on the Akaike information criterion (AIC) (Akaike 1974) and the Bayesian information criterion (BIC) (Schwarz 1978). For both criteria a lower value indicates a better model fit, while accounting for overfitting. In the case that the AIC and BIC values did not agree on the same model, the value of the BIC was used to select a statistical model for further analyses.

The first model assumed that neurophysiological activity of both pulses was integrated by temporal summation, where the neurophysiological activity generated as a result of the first pulse amplitude (in mA, denoted by PU1) is summed with activity generated as a result of the second pulse amplitude with either $10 \mathrm{~ms}$ IPI (in mA, denoted by PU2 ${ }_{10}$ ) or $40 \mathrm{~ms}$ IPI (in $\mathrm{mA}$, denoted by PU $2_{40}$ ). Furthermore, this signal could decrease with respect to the trial number (denoted by TRL) due to habituation effects.

The resulting generalized linear mixed regression model for computing detection probability in A was compared to models using a direct combination of the experimental parameters in $\mathrm{B}, \mathrm{C}$ and $\mathrm{D}$, where the response was modeled based on the stimulus amplitude (in mA, denoted by AMP), stimulus type (denoted by TYP) and the trial number. The random effect structure was grouped by subject (denoted by S) and included all model terms. An unstructured covariance structure was used to model the random effects. Models are written in Wilkinson notation (Wilkinson and Rogers 1973), where random effects are written in between brackets and ' $S$ ' denotes that the random effects are grouped by subject.

$$
\begin{aligned}
\ln \left(\frac{P_{d}}{1-P_{d}}\right) \sim 1 & +P U 1+P U 2_{10}+P U 2_{40}+T R L \\
& +\left(1+P U 1+P U 2_{10}+P U 2_{40}+T R L \mid S\right)
\end{aligned}
$$

$\ln \left(\frac{P_{d}}{1-P_{d}}\right) \sim 1+A M P * T Y P * T R L+(1+A M P * T Y P * T R L \mid S)$

$\ln \left(\frac{P_{d}}{1-P_{d}}\right) \sim 1+A M P * T Y P+T R L+(1+A M P * T Y P+T R L \mid S)$

$\ln \left(\frac{P_{d}}{1-P_{d}}\right) \sim 1+A M P+T Y P+T R L+(1+A M P+T Y P+T R L \mid S)$

The first linear mixed regression model for analyzing EEG activity was based on the temporal summation model in A, but included a term for additional brain activity evoked by stimulus detection which could increase or decrease with respect to the trial number (denoted by the interaction TRL*D). The resulting model in $\mathrm{E}$ was compared to the models based on combinations of experimental parameters in F, G, H and I at the P2 latency (414 ms). The random effect structure was grouped by subject and included all model terms. A diagonal covariance structure was used to model the random effects.

$$
\begin{aligned}
U_{E E G} \sim 1 & +P U 1+P U 2_{10}+P U 2_{40}+T R L * D \\
& +\left(1+P U 1+P U 2_{10}+P U 2_{40}+T R L * D \mid S\right)
\end{aligned}
$$

$U_{E E G} \sim 1+A M P * T Y P * T R L * D+(1+A M P * T Y P * T R L * D \mid S)$

$U_{E E G} \sim 1+A M P * T Y P * D+T R L * D$

$$
+(1+A M P * T Y P * D+T R L * D \mid S)
$$

$U_{E E G} \sim 1+A M P * T Y P+T R L * D+(1+A M P * T Y P+T R L * D \mid S)$

$U_{E E G} \sim 1+A M P+T Y P+T R L+D+(1+A M P+T Y P+T R L+D \mid S)$

\section{Results}

\section{Model Selection}

A functional model was compared to models directly based on the experimental parameters for statistical analysis of the detection probability and the EEG. Model AIC and BIC values are shown in Table 1. For the detection probability, the experiment-based model B including all effects resulted in the lowest AIC. For EEG data, the functional model E resulted in 
Table 1 AIC and BIC values for comparison of the functional models (A and E) with various experiment-based models

\begin{tabular}{lllllll}
\hline \multicolumn{2}{l}{ Detection } & probability & & & \multicolumn{2}{l}{ EEG } \\
\cline { 1 - 1 } Model & AIC & BIC & & Model & AIC & BIC \\
\hline A & 13,444 & $\mathbf{1 3 , 5 9 1}$ & & E & $\mathbf{2 5 , 0 6 9}$ & $\mathbf{2 5 , 1 7 7}$ \\
B & $\mathbf{1 3 , 3 9 3}$ & 14,053 & & F & 25,389 & 25,575 \\
C & 13,413 & 13,670 & & G & 25,107 & 25,314 \\
D & 14,015 & 14,161 & & H & 25,105 & 25,241 \\
& & & I & 25,122 & 25,215 \\
\hline
\end{tabular}

A lower AIC or BIC value indicates a better model fit while accounting for the number of parameters in the model. Values in bold indicate the model with the lowest AIC or BIC

the lowest AIC. For both the detection probability and the EEG data, the functional models $\mathrm{A}$ and $\mathrm{E}$ resulted in the lowest BIC.

\section{Effect of Intra-Epidermal Stimulus Properties on Detection Probability}

Subjects detected on average $46.7 \%$ of all applied stimuli with a reaction time of $546 \pm 161 \mathrm{~ms}$. A typical example of a resulting tracked NDT in a single subject is shown on the left side of Fig. 3. For each stimulus type, the NDT increased over time. Both thresholds for double pulse stimuli

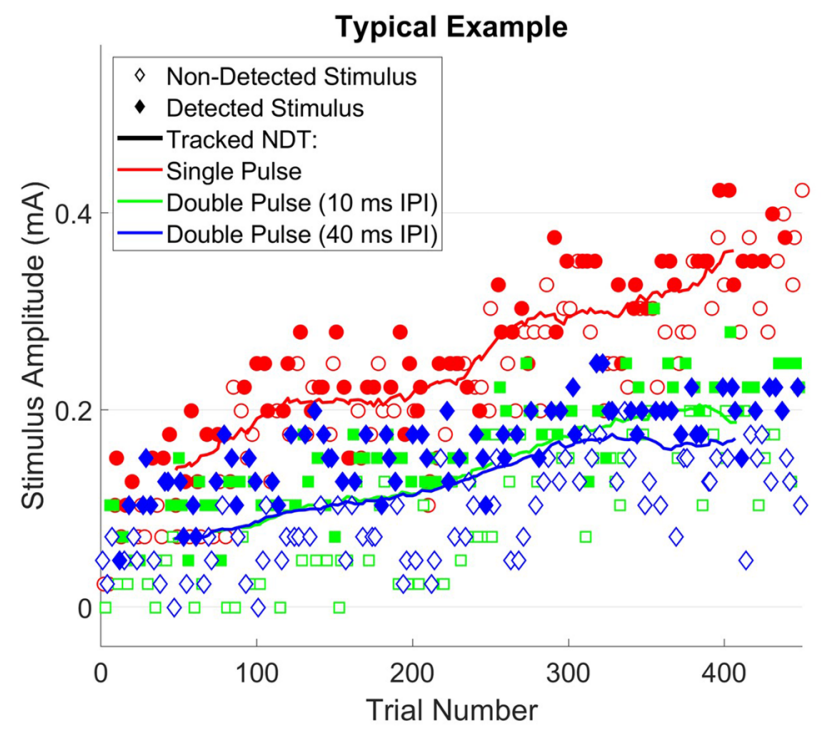

Fig. 3 A typical example of tracked nociceptive detection thresholds (NDTs) (left) and group level NDTs (right). Both group level detection thresholds computed over a time window of 30 stimuli (tracked NDT) and computed over the entire experiment (GLMR NDT) are shown. The standard error of the mean (SEM) of tracked thresholds were almost equal, i.e. there was no difference of NDT with respect to the inter-pulse interval in this subject. Similar results are shown in the group level thresholds on the right in Fig. 3, computed using the GLMR model over a 30 trial moving window (continuous lines) and over the entire dataset (dotted lines). Group level NDTs computed over the entire dataset increased over the trials and remained within the standard error of the mean (SEM) of NDTs computed over a 30 trial moving window. Once again, no difference was seen between NDTs of double pulse stimuli with $10 \mathrm{~ms}$ inter-pulse interval and those with $40 \mathrm{~ms}$ inter-pulse interval.

Observations in Fig. 3 are supported by effect sizes and significances in Table 2. There was a significant positive effect size for each of the pulse amplitudes, indicating an increase in detection probability with respect to the pulse amplitudes. There was a significant negative effect size of trial number, indicating a decrease of detection probability with respect to the trial number. In Table 3 , it is shown that there was indeed a significantly lower detection threshold $(p<0.001)$ and steeper slope $(p<0.001)$ for both types of double-pulse stimuli in comparison with a single-pulse stimulus. However, there was no significant difference in detection threshold or slope between double pulse stimuli with 10 or $40 \mathrm{~ms}$ inter-pulse interval.

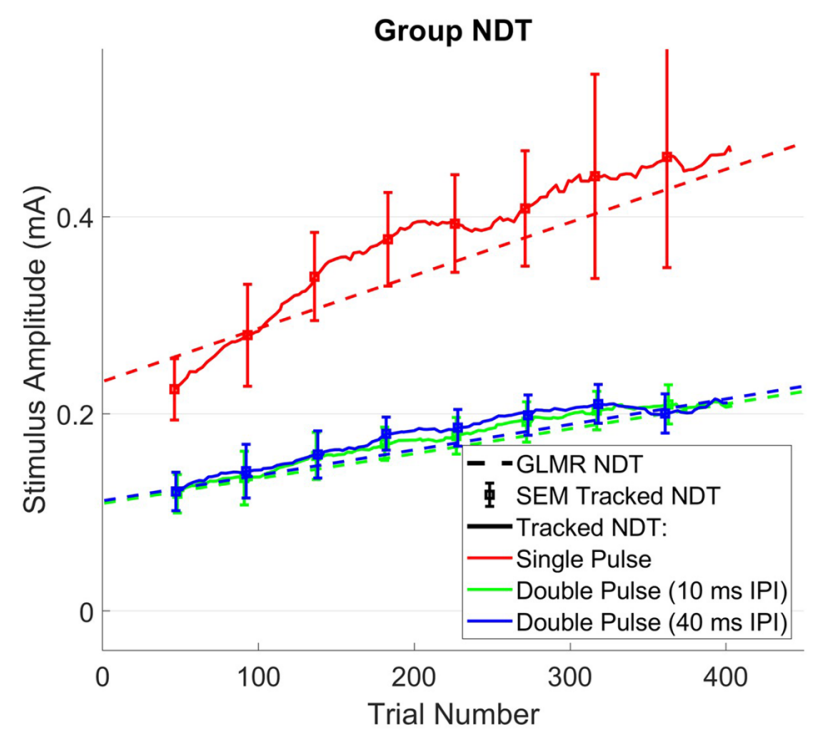

is indicated by bars. For each NDT, it can be observed that the threshold increased over time and that the threshold for double pulse stimuli was much lower. However, there was no difference in detection threshold with respect to inter-pulse interval (IPI) 
Table 2 Effect of stimulus properties on the detection probability, computed using GLMR
Table 3 Detection thresholds (in $\mathrm{mA}$ ) and slopes (in $\mathrm{mA}^{-1}$ ) per stimulus type

\begin{tabular}{lclrr}
\hline Stimulus property & Effect size & $95 \%$ Confidence interval & Effect $\chi^{2}$ & Effect $p$ \\
\hline (Intercept) & -3.70 & {$[-4.32,-3.09]$} & 139.61 & $<.001$ \\
Pulse 1 (PU1) & 10.43 & {$[7.14,13.72]$} & 38.67 & $<.001$ \\
Pulse 2, 10 ms IPI (PU2 $\left.{ }_{10}\right)$ & 11.83 & {$[9.03,14.63]$} & 68.38 & $<.001$ \\
Pulse 2, 40 ms IPI (PU2 40$)$ & 11.31 & {$[8.61,14.01]$} & 67.40 & $<.001$ \\
Trial number (TRL) & -0.0056 & {$[-0.0074,-0.0038]$} & 37.69 & $<.001$ \\
\hline
\end{tabular}

All effect sizes and confidence intervals were rescaled to physical units (Int.: -, PU1: $\mathrm{mA}^{-1}$, PU2 ${ }_{10}: \mathrm{mA}^{-1}$, PU2 $2_{40}: \mathrm{mA}^{-1}$, TRL: trial $^{-1}$ ). Significance was assessed using type-III Wald Chi-square statistics. All tested stimulus properties have a significant effect on the detection probability. The detection probability decreases with respect to trial number and increases with respect to the amplitude of the first pulse and of the second pulse with either $10 \mathrm{~ms}$ or $40 \mathrm{~ms}$ IPI

\begin{tabular}{lllll}
\hline Stimulus Type & Threshold & $\begin{array}{l}\text { 95\% Confidence } \\
\text { Interval }\end{array}$ & Slope & 95\% Confidence Interval \\
\hline Single-pulse & 0.35 & {$[0.33,0.52]$} & 10.43 & {$[7.20,13.66]$} \\
Double-pulse, 10 ms IPI & $0.17^{* * *}$ & {$[0.14,0.20]$} & $22.26^{* * *}$ & {$[17.15,27.37]$} \\
Double-pulse, 40 ms IPI & $0.17 * * *$ & {$[0.14,0.20]$} & $21.74^{* * *}$ & {$[16.42,27.06]$} \\
\hline
\end{tabular}

There is a significantly $(\mathrm{p}<0.001$, indicated by $* * *)$ lower detection threshold and steeper slope for each type of double-pulse stimulus in comparison to the detection threshold of single-pulse stimuli. There was no significant difference in detection threshold or slope between both types of double-pulse stimuli
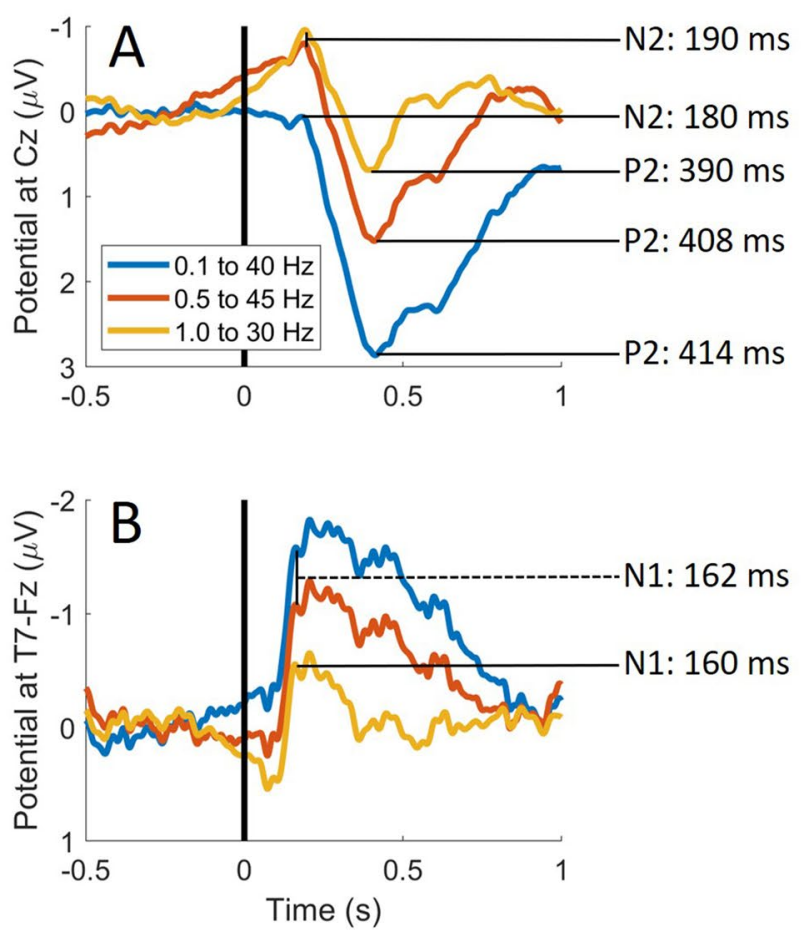
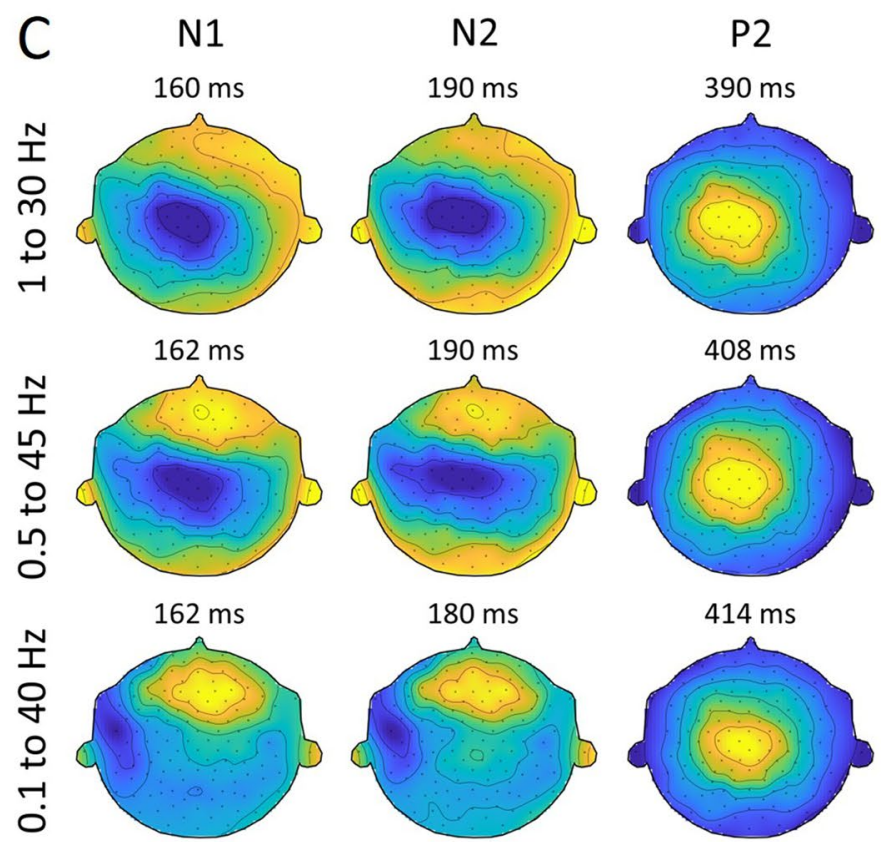

Fig. 4 a, b Evoked potential at $\mathrm{Cz}$ (average reference) and T7-Fz band-pass filtered at 0.1 to $40 \mathrm{~Hz}, 0.5$ to $45 \mathrm{~Hz}$ and 1 to $30 \mathrm{~Hz}$, and latencies of the peaks in each of the EP waveforms. c Evoked potential topographies at the latencies of N1, N2 and P2 


\section{Evoked Potential in Response to Intra-Epidermal Stimuli}

For comparison with previously observed EP waveforms in response to intra-epidermal stimulation in literature (Liang et al. 2016; Mouraux et al. 2014), EP waveforms at the same channels and with the same filters as in those studies are shown in Fig. 4a, b. The N1 was located at $160 \mathrm{~ms}$ (1 to $30 \mathrm{~Hz})$ and $162 \mathrm{~ms}(0.1$ to $40 \mathrm{~Hz}$ and 0.5 to $45 \mathrm{~Hz})$ at T7-Fz. The N2, was located at $180 \mathrm{~ms}(0.1$ to $40 \mathrm{~Hz})$ and $190 \mathrm{~ms}$ $(0.5$ to $45 \mathrm{~Hz}$ and 1 to $30 \mathrm{~Hz}$ ) at $\mathrm{Cz}$. The $\mathrm{P} 2$ was located at $390 \mathrm{~ms}$ (1 to $30 \mathrm{~Hz}), 408 \mathrm{~ms}(0.5$ to $45 \mathrm{~Hz})$ and $414 \mathrm{~ms}(0.1$ to $40 \mathrm{~Hz}$ ).
Grand average EP topographies at these peak latencies are displayed in Fig. 4c. Topographies of the N1 and N2 were similar to each other for all filter settings, but differ per filter setting. The $\mathrm{N} 1$ and $\mathrm{N} 2$ both showed a central negative topography when using filters of 0.5 to $45 \mathrm{~Hz}$ and of 1 to $30 \mathrm{~Hz}$. In contrast, the $\mathrm{N} 1$ and $\mathrm{N} 2$ showed a distinct contralateral topography when using filters of 0.1 to $40 \mathrm{~Hz}$. The topography of the $\mathrm{P} 2$ was central and positive for all filter settings.

A butterfly plot of grand average EPs and the global field power (GFP) in response to the intra-epidermal electric stimuli is shown in Fig. 5. The global field power showed a major peak around the $\mathrm{P} 2$ and a minor peak around the $\mathrm{N} 1$,
Fig. 5 Butterfly plot of the grand average potential and global field power (GFP) of EEG channels in response to intra-epidermal stimuli around the nociceptive detection threshold $(<2 \times$ NDT $)$. The N1 appeared to coincide with an early peak of the GFP, while the $\mathrm{P} 2$ coincided with the maximum of the GFP. The N2 did not coincide with any peak in the GFP or in the butterfly plot

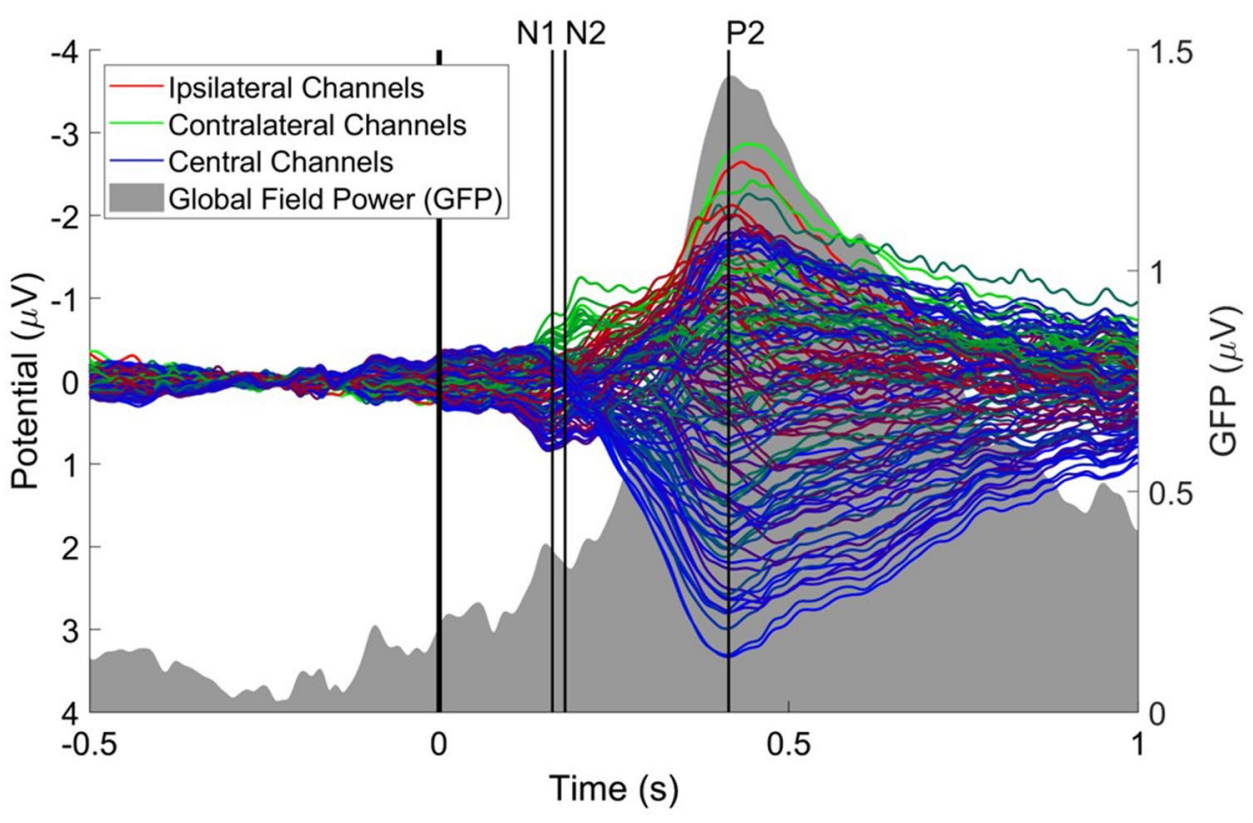

Table 4 Grand average electrode potential and SNR at N1, N2 and P2

\begin{tabular}{|c|c|c|c|c|c|c|c|c|c|}
\hline & N1 Channel & Value $(\mu \mathrm{V})$ & SNR & N2 Channel & Value $(\mu \mathrm{V})$ & SNR & P2 Channel & Value $(\mu \mathrm{V})$ & SNR \\
\hline 1 & $\mathrm{~F} 4 *$ & 0.83 & 23.49 & $\mathrm{~T} 7 *$ & -0.82 & 18.36 & $\mathrm{CPz}^{* *}$ & 3.33 & 185.57 \\
\hline 2 & $\mathrm{~T} 7 *$ & -0.82 & 18.22 & $\mathrm{~F} 4 *$ & 0.77 & 21.76 & $\mathrm{CCP} 1 \mathrm{~h} * * *$ & 3.31 & 122.68 \\
\hline 3 & $\mathrm{FFC} 2 \mathrm{~h} * * *$ & 0.81 & 17.41 & $\mathrm{~F} 1 * *$ & 0.75 & 14.41 & $\mathrm{CP} 1 * *$ & 2.99 & 111.57 \\
\hline 4 & $\mathrm{~F} 2 * *$ & 0.79 & 20.35 & $\mathrm{~F} 2 * *$ & 0.72 & 18.76 & $\mathrm{Cz}^{*}$ & 2.80 & 97.37 \\
\hline 5 & $\mathrm{Fz}^{*}$ & 0.77 & 18.96 & $\mathrm{FFC} 2 \mathrm{~h} * * *$ & 0.72 & 15.42 & $\mathrm{CCP} 2 \mathrm{~h} * * *$ & 2.78 & 163.37 \\
\hline 6 & FFC4h*** & 0.71 & 15.24 & TTP7h*** & -0.71 & 20.61 & $\mathrm{C} 1 * *$ & 2.76 & 91.46 \\
\hline 7 & $\mathrm{~F} 1 * *$ & 0.71 & 13.65 & $\mathrm{Fz}^{*}$ & 0.70 & 17.12 & $\mathrm{~A} 1 *$ & -2.74 & 36.57 \\
\hline 8 & $\mathrm{AFF} 2 * * *$ & 0.69 & 19.38 & $\mathrm{AFz}^{* * *}$ & 0.67 & 13.67 & $\mathrm{CPP} 2 \mathrm{~h} * * *$ & 2.64 & 111.59 \\
\hline 9 & $\mathrm{FC} 2 *$ & 0.68 & 17.85 & FT9 $9 * *$ & -0.63 & 12.16 & CPP1h*** & 2.62 & 92.24 \\
\hline 10 & $\mathrm{AFz}^{* * *}$ & 0.68 & 13.88 & $\mathrm{AFF} 2 * * *$ & 0.63 & 17.81 & CCP $3 h^{* * * *}$ & 2.57 & 91.15 \\
\hline 11 & FT9*** & -0.64 & 12.24 & FFC4h*** & 0.62 & 13.44 & $\mathrm{~A} 2 *$ & -2.56 & 30.74 \\
\hline 12 & TTP7h*** & -0.63 & 18.36 & $\mathrm{FC} 2 *$ & 0.59 & 15.44 & $\mathrm{CP} 2 *$ & 2.51 & 126.85 \\
\hline
\end{tabular}

The electrodes with the largest potential values were also the electrodes with the largest SNR at N1 (F4) and P2 (CPz). Electrodes which are present in a regular 32-channel cap, 64-channel cap and 128-channel cap (10-5 system) are denoted with *, ** and *** respectively 


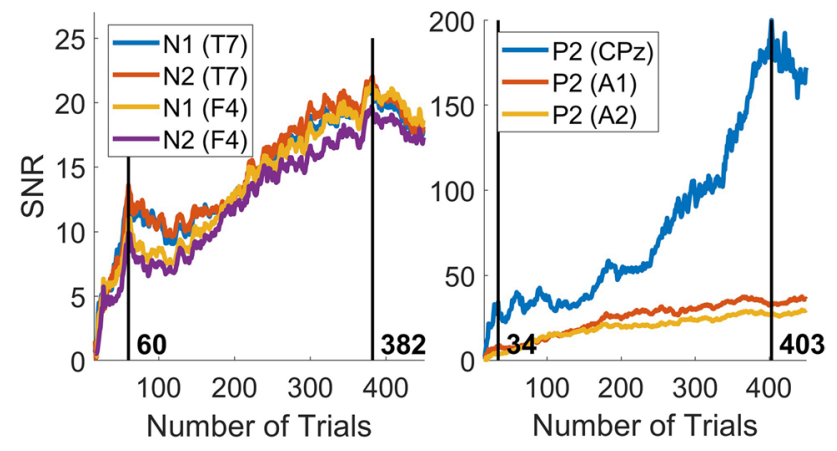

Fig. 6 Signal-to-noise ratio (SNR) of the evoked potential in the grand average of the channels $\mathrm{T} 7$ and $\mathrm{F} 4$ (for $\mathrm{N} 1$ and $\mathrm{N} 2$ ), and $\mathrm{CPz}$, $\mathrm{A} 1$ and $\mathrm{A} 2$ (for P2). For N1 and N2, there is an initial peak of the SNR after 60 trials, and a maximum at 382 trials. For P2, there is an initial peak of the SNR after 34 trials and a maximum at 403 trials

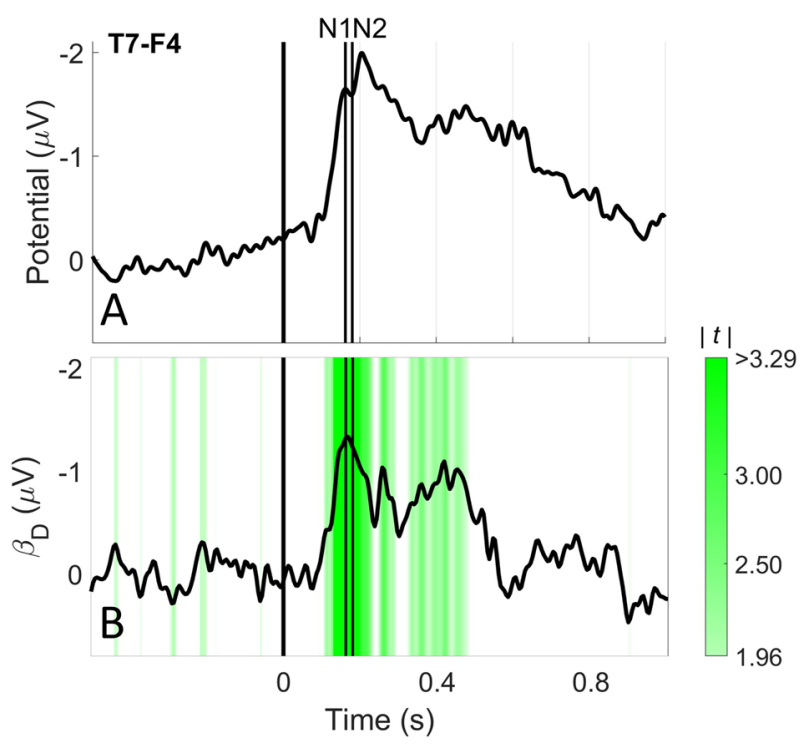

Fig. 7 Grand average EP $\mathbf{a}$ and the effect of stimulus properties $\mathbf{b}$ at T7-F4. The corresponding t-values are shown in green on a scale of $1.96(\mathrm{p}=0.05$ with inf. DOF) to $3.29(\mathrm{p}=0.001$ with inf. DOF). At T7-F4 only stimulus detection had a significant effect on the EP at the $\mathrm{N} 1$ and N2 latencies

each corresponding to local maxima in a subset of channels. However, the N2 did not appear to coincide with any peak of global field power or of any subset of channels.

For each latency, the 12 channels with the largest positive and negative potentials are shown in Table 4. In addition, the SNR of each channel was computed, which in this case correlated strongly with the potential (i.e. the channels with the largest potential also have a large SNR). The derivation with the largest potential at each latency was found by subtracting the most negative channel(s) from the most positive channel or vice-versa depending on polarity. Based on these considerations, T7-F4 and CPz-A1A2 were selected for further investigation of the EP.

For the selected channels, the SNR is shown as a function of the number of trials in Fig. 6. For the N1 and N2, there is an initial peak of SNR after 60 trials. For the P2, there is an initial peak of the SNR after 34 trials. However, in both cases the SNR tends to increase with an increasing number of trials, with a maximum at 382 trials for the N1 and N2, and a maximum at 403 trials for the P2.

\section{Effect of Intra-Epidermal Stimulus Properties on Evoked Potential}

The grand average EP at T7-F4 and CPz-A1A2 is shown in Figs. 7a and 8a respectively. At T7-F4 intra-epidermal stimuli elicited an early negative component with a peak around the N1 latency, followed by a small positive component. At CPz-A1A2, intra-epidermal stimuli elicited a clear positive component with a maximum close to the $\mathrm{P} 2$ latency.

The results of significance testing of stimulus properties at T7-F4 and CPz-A1A2 are shown in Table 5. At N1 and $\mathrm{N} 2$ only stimulus detection was significant. At P2 stimulus detection, each pulse amplitude and the interaction between detection and trial number were significant. For all significant effects, the effect sizes and $t$-values over time are displayed in Fig. 7b (for T7-F4) and 8B-F (for CPz-A1A2). For T7-F4, the negative effect of detection was mostly concentrated around the N1. For CPz-A1A2, the positive effect of each pulse amplitude started before the P2 and lasted for several hundred milliseconds. The interaction between detection and trial number had a negative effect during the same time range as the pulse amplitudes. Detection had a positive effect starting before the $\mathrm{P} 2$ and lasting until the end of the epoch.

\section{Discussion}

We have simultaneously assessed neurophysiological and psychophysical effects of nociceptive intra-epidermal stimulation using a method to simultaneously measure EPs and detection thresholds in response to multiple stimulus types. Preferential activation of nociceptive afferents was achieved by stimulating at intensities close to the detection thresholds, and excluding trials from EEG analysis if the stimulus was larger than twice the NDT (Mouraux et al. 2010). We aimed to confirm the presence, location and latency of EP components and to quantify the effect of intra-epidermal stimulus properties on those components. 


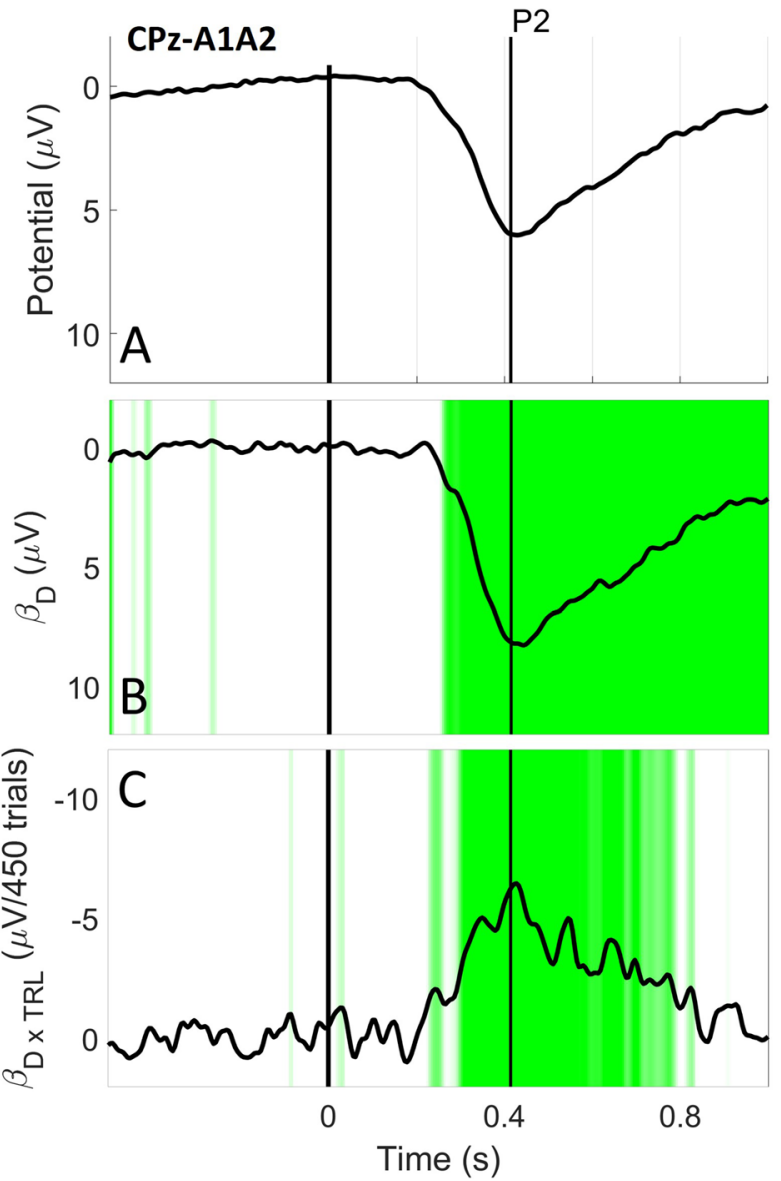

Fig. 8 Grand average EP $\mathbf{a}$ and the effect of stimulus properties $\mathbf{b}-\mathbf{f}$ at $\mathrm{CPz}-\mathrm{A} 1 \mathrm{~A} 2$. The corresponding t-values are shown in green on a scale of $1.96(p=0.05$ with inf. DOF) to $3.29(p=0.001$ with inf. DOF). At CPz-A1A2 stimulus detection, the amplitude of each pulse and the

\section{Evoked Potential in Response to Intra-Epidermal Stimuli}

The EP waveforms observed in this study correspond with those observed in previous studies using intra-epidermal stimulation (Fig. 4a, b). Where an earlier study by Mouraux et al. (2014) observed a N1 component in response to intra-epidermal stimulation around a latency of $150 \mathrm{~ms}$, we observed a $\mathrm{N} 1$ component at the same derivation $(\mathrm{T} 7-\mathrm{Fz})$ and with the same filter settings around 160 ms. Furthermore, Mouraux et al. observed a N2 component at $\mathrm{Cz}$ in the range of $220-230 \mathrm{~ms}$ and another study by Liang et al. (2016) observed a much earlier N2 component at the same channel in the range of $130-150 \mathrm{~ms}$. The observed $\mathrm{N} 2$ component in the current study occurs at the same channel and filter setting at a latency in between those estimates, around $190 \mathrm{~ms}$. For both the N1 and N2 the largest positive and negative potential values and SNR were found at F4 and T7 respectively. The SNR of both

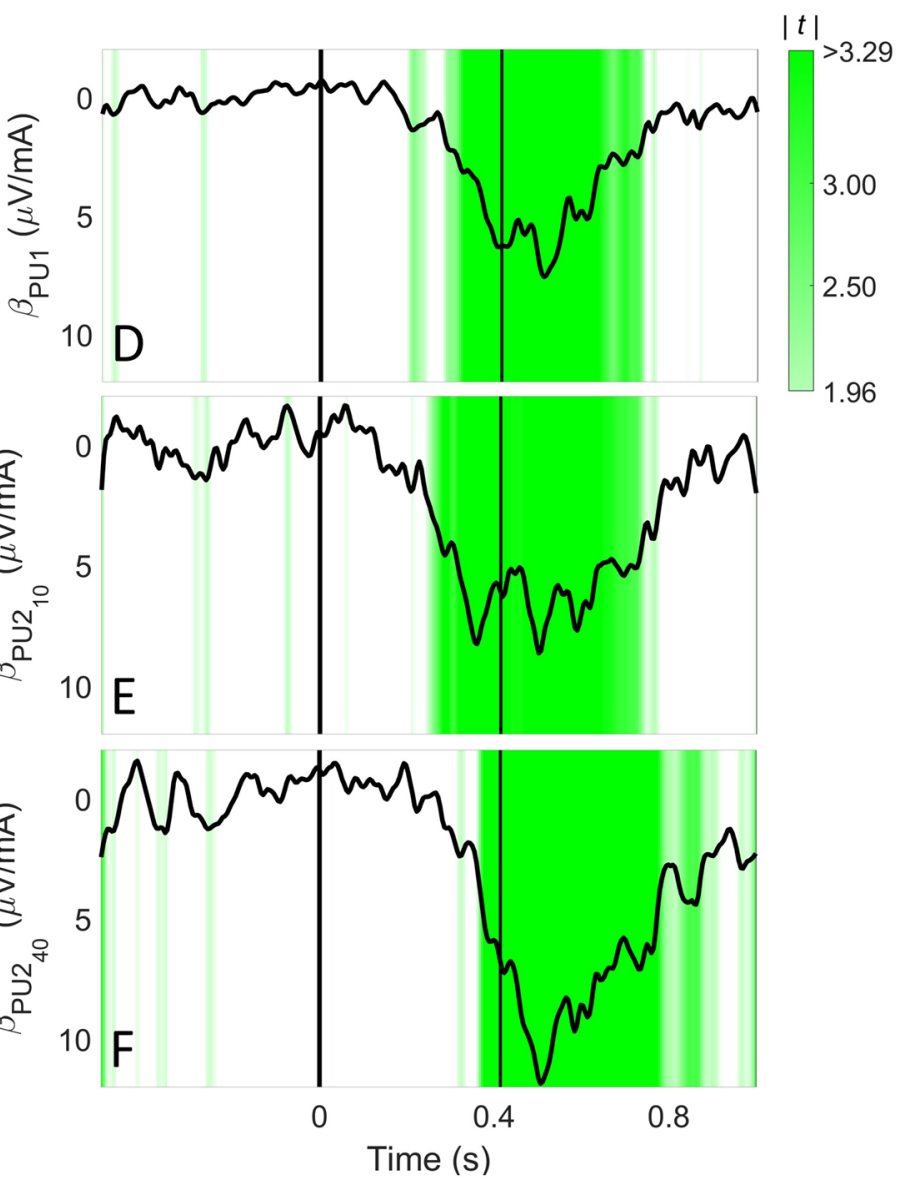

interaction between detection and trial number had a significant effect on the EP at the P2 latency which lasted for several hundreds of milliseconds

components at $\mathrm{T} 7$ and $\mathrm{F} 4$ was shown to increase with respect to the number of trials included in the grand average. However, after a steep increase of the SNR within the first 60 trials, the improvement of SNR flattens, potentially due to habituation affects and loss of attention.

The observed latencies of the $\mathrm{P} 2$ at $\mathrm{Cz}$ of 390 and $408 \mathrm{~ms}$, are slightly later than those observed by Liang et al. (290-330 ms) and Mouraux et al. (360-370 ms) respectively. Although the observed amplitude is much lower than in studies using heat pulses (Miyazaki et al. 1994; Treede et al. 1988), it is comparable to earlier nociceptive EP studies using intra-epidermal stimulation near the detection threshold (Mouraux et al. 2010; Van der Lubbe et al. 2017), indicating that this difference in potential might be due to stimulus intensity. The potential values in Table 4 show that during the observed P2, the largest positive potential value (and SNR) could be found at $\mathrm{CPz}$ and the lowest negative potential value (and SNR) could be found at A1 followed by A2. The SNR of the P2 at CPz, 


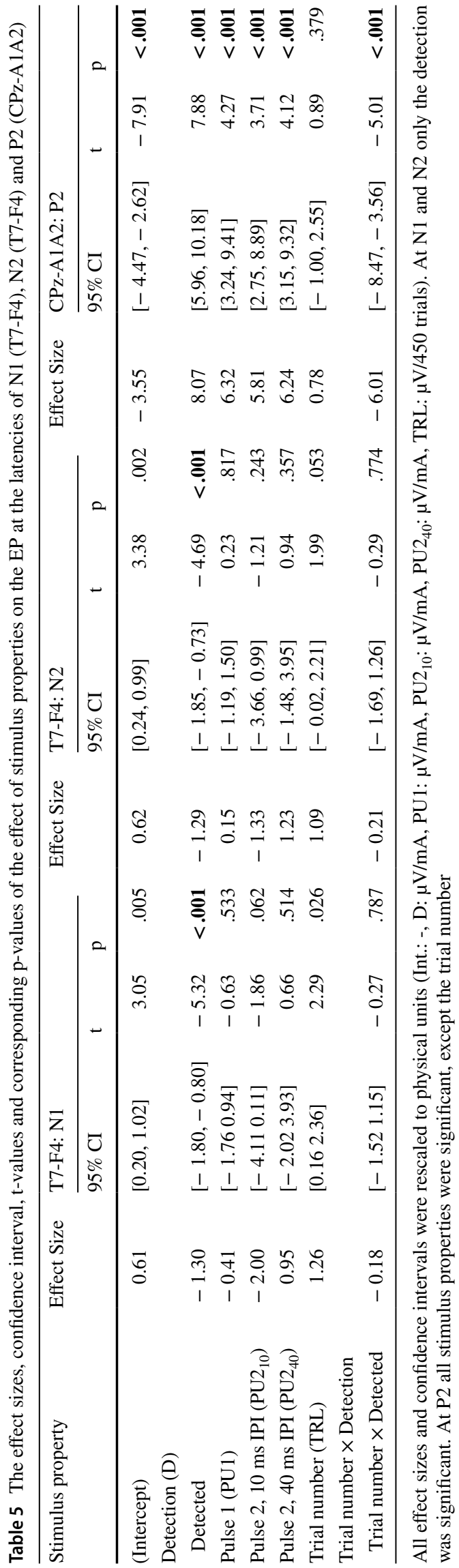

A1 and A2 improved the most within the first 34 trials, but kept increasing steeply until reaching a maximum close to the end of the experiment at 403 trials.

Although the observed EP waveform corresponds to previous studies using similar derivations and filter setting, it is clear from Fig. 4 that filter settings have a profound influence on the EP waveform and topography. While the choice of a larger cutoff frequency for the high-pass filter enhances the $\mathrm{N} 2$ component at $\mathrm{Cz}$, it strongly decreases amplitude of the P2. Furthermore, the filter setting has a profound influence on the observed topographies of N1 and N2. While the topographies of $\mathrm{N} 1$ and $\mathrm{N} 2$ in this study show a maximum around the vertex with a high-pass filter of 0.5 or $1 \mathrm{~Hz}$, both topographies have a maximum contralateral to stimulation when using a high-pass filter of $0.1 \mathrm{~Hz}$. In this work, we chose to minimize high-pass filter signal distortion, by choosing a relatively low high-pass filter cutoff frequency of $0.1 \mathrm{~Hz}$ (Acunzo et al. 2012).

When using a low high-pass filter cutoff frequency, the P2 amplitude is increased, but the N2 amplitude is severely reduced. Using this filter setting the observed global field power showed peaks only around the $\mathrm{N} 1$ latency and the $\mathrm{P} 2$ latency. Topographies of the N1 and N2 are almost identical, with a maximum at $\mathrm{T} 7$ and a minimum at F4. As such, it is questionable whether N1 and N2 identified in this study really represent independent components of brain activity. In the current study, it appears that the N2 rather arose from high-pass filter settings rather than physiological activity, as the high-pass filter essentially works as a signal differentiator resulting in peaks of opposite polarity before occurrence of the true effect, i.e. the P2 (Tanner et al. 2015).

\section{Effect of Intra-Epidermal Stimulus Properties on Detection Probability}

Intra-epidermal electric stimuli directly activate superficial afferents in the skin, rather than activating skin receptors (Inui and Kakigi 2012; Inui et al. 2002). As this signal is transduced by the relatively slow $A \delta$-fibers, rather than the fast $A \beta$-fibers, the reaction times to this type of stimulation are usually increased with respect to conventional transcutaneous electric stimulation. Correspondingly, the average reaction time in this study $(546 \pm 161 \mathrm{~ms})$ was markedly later than previously observed reaction times (Mouraux et al. 2010) to transcutaneous stimulation $(283 \pm 47 \mathrm{~ms})$ and intra-epidermal stimulation at twice the perceptual threshold $(374 \pm 51 \mathrm{~ms})$, but similar to laser stimulation $(504 \pm 105 \mathrm{~ms})$. As such, reaction times suggest preferential recruitment of A $\delta$-fibers.

Increasing the pulse amplitude directly enlarges the area of recruitment of peripheral afferent nerve fibers (Poulsen et al. 2020). Increasing the number of pulses results in the generation of more action potentials. Central synaptic 
summation of these action potentials occurs in the spinal cord, where facilitatory or inhibitory effects could occur depending on the inter-pulse interval (Zucker and Regehr 2002). Nociceptive processing adapts to repeated stimulus application, leading to a habituation of neurophysiological (Christmann et al. 2007) and psychophysical (May et al. 2012) responses. One of the aims was to probe each of these mechanisms by varying pulse amplitudes, the number of pulses and the inter-pulse interval of the applied stimuli. We started out by formulating and comparing multiple statistical models to study the effect of these stimulus properties. This included functional models explaining detection probability and EEG in terms of pulse amplitudes and trial number. It was found in Table 1 that both functional models (A and E) have the lowest BIC, indicating that among the tested models these models are the closest approximation of the true physiological behavior.

We found that nociceptive stimulus detection behaves according to theory, where the pulse amplitudes and trial number have a significant effect on the detection probability. The effects of pulse amplitudes observed in this study lie within the confidence interval reported in the earlier technical demonstration of the method (van den Berg et al. 2020). As was expected based on the larger recruitment of peripheral afferents, the detection probability increased with increasing pulse amplitudes. Furthermore, we saw that addition of a second pulse with either $10 \mathrm{~ms}$ or $40 \mathrm{~ms}$ inter-pulse interval leads to a significant increase of detection probability (Fig. 3; Table 2) similar to earlier observations by Doll et al. (2016a, b). This results in significantly lower detection thresholds and significantly steeper slopes for these double pulse stimuli (Table 3). However, there is no difference between double-pulse detection thresholds and slopes dependent on the inter-pulse interval, which was either 10 or $40 \mathrm{~ms}$. Experiments in humans subjects measuring the compound sensory action potential in response to paired pulses indicate that peripheral sensory nerve fibers remain superexcitable up to approximately $20 \mathrm{~ms}$ after the first pulse and remain subexcitable from 20 to $100 \mathrm{~ms}$ after the stimulus (Kiernan et al. 1996). If these findings also hold for nociceptive afferents, one would expect a higher detection probability and lower threshold for double-pulse stimuli with an inter-pulse interval of $10 \mathrm{~ms}$ than for double-pulse stimuli with an inter-pulse interval of $40 \mathrm{~ms}$. It turns the effect of adding a second-pulse is similar regardless of the inter-pulse interval, resulting in similar detection thresholds for both stimulus types. As such, the effects of peripheral super- and subexcitability appear to be canceled out by a stronger central mechanism, such as the central temporal summation of both pulses (Zucker and Regehr 2002).

The detection probability decreases over the number of trials, resulting in an increase of the detection threshold in Fig. 3. In earlier studies, this effect was also found significant, but had a larger effect size (Doll et al. 2016a, b; van den Berg et al. 2020). Altered habituation appears to play an important role in several types of chronic pain syndromes, and is therefore an important phenomenon to observe when assessing nociceptive processing (Agostinho et al. 2009; Rodriguez-Raecke et al. 2014; Valeriani et al. 2003). Nevertheless, the neurophysiological mechanisms of this effect remain unknown, and this effect might be attributed to either an altered task performance, a shift of attention, learning or neuroplasticity.

\section{Effect of Intra-Epidermal Stimulus Properties on Evoked Potential}

The effect of stimulus properties on the EP was shown in Table 5. Based on Figs. 7b and 8b-f we could also observe at which latencies these effect sizes were largest. We did not find that the lateral potential at T7-F4 behaves according to the theory mentioned earlier. Instead, it was only significantly modulated by detection with its major effect size around the latency of N1. As such, we did not observe any significant encoding of physical properties of the stimulus (i.e. the pulse amplitudes) in the N1 or N2. It remains unknown if this absence of the effect of stimulus properties on lateral EP components is because these components do not encode any physical properties of a stimulus, or simply because the $\mathrm{N} 1$ and $\mathrm{N} 2$ are relatively small signals and easily obscured by background noise.

The potential at CPz-A1 A2 around at P2 latency was not only modulated by detection, but also by the pulse amplitudes and the interaction between detection and trial number. The latter observation is consistent with earlier reports in literature, which show that the $\mathrm{P} 2$ represents multi-modal activity dependent on stimulus salience (Iannetti et al. 2008; Legrain et al. 2011). As the pulse amplitudes and the trial number influence stimulus salience through the mechanisms discussed in last section, these properties were also expected to affect the P2. We saw that the contribution of the second pulse is equal or even larger than the contribution of the first pulse to the $\mathrm{P} 2$. This corresponds to the observation in last section, where the detection threshold is lowered by the addition of a second pulse and the effect size of both types of second pulse is actually larger than the effect size of the first pulse amplitude. As such, the observed increase in P2 amplitude is likely to be associated with similar facilitatory mechanisms as discussed in "Effect of Intra-Epidermal Stimulus Properties on Detection Probability" section.

We also observed a significant effect of the interaction between detection and trial number on the $\mathrm{P} 2$ amplitude. As such, the P2 decreases with respect to trial number, but only in response to detected stimuli. Measuring this habituating behavior can be used to assess altered nociceptive processing. A decreased habituation of $\mathrm{P} 2$ amplitude over 
time or over the amount of repeated stimuli has been related to chronic pain in earlier literature in patients with migraine (Valeriani et al. 2003), chronic low back pain (Vossen et al. 2015) and fibromyalgia (de Tommaso et al. 2011).

\section{Conclusion}

After a technical demonstration of combined threshold tracking and EP acquisition in an earlier study (van den Berg et al. 2020), we started this study to determine (1) which EP components can be observed during this procedure, (2) at which scalp locations these components are best observed and (3) to quantify the effect of stimulus properties on these components and detection thresholds in healthy subjects. We found that an N1 and N2 component can be observed with a maximum positive and negative potential at $\mathrm{F} 4$ and $\mathrm{T} 7$ respectively. The $\mathrm{P} 2$ component can be observed with maximum positive and negative potentials at $\mathrm{CPz}$ and $\mathrm{A} 1$ respectively. The $\mathrm{P} 2$ has a similar latency and topography regardless of filter settings. However, the N1 and N2 waveform and topography are heavily affected by the high-pass cutoff frequency. Using a larger cutoff frequency enhanced the N2 and shifted the topographies of N1 and N2 from contralateral to central, suggesting that the observed N2 could be an artifactual effect of high-pass filtering. Statistical analysis showed that the $\mathrm{N} 1$ and $\mathrm{N} 2$ components observed in this experiment mainly influence by stimulus detection, while the $\mathrm{P} 2$ as well as the detection probability of a stimulus are also significantly influenced by stimulus properties such as the pulse amplitudes and the trial number.

Measuring the effects of intra-epidermal stimulus properties on the detection threshold and the evoked potential simultaneously provides a way to measure brain activation and pain perception in response to a well-defined nociceptive input. The results in this study demonstrate that the various steps of processing of a nociceptive stimulus, including peripheral nerve fiber recruitment, central synaptic summation, and habituation to a repeated stimulus are reflected by the detection thresholds as well as the EP.

Funding This study was funded by the Dutch Research Council (NWO) through the NeuroCIMT research program (P14-12, project 2).

Data Availability A limited dataset of the experiments reported here is available on request.

Code Availability Code required to perform the analyses reported here is available on request.

\section{Compliance with Ethical Standards}

Conflict of interest The authors declare that they have no conflict of interest.

Consent to Participate All participants provided written informed consent and were rewarded for participation in the experiment.

Consent for Publication All authors approved the manuscript and agree with submission to Experimental Brain Research.

Ethical Approval All experiments were approved by the Medical Review and Ethics Committee (METC Twente, ABR: NL62721.044.17) and in accordance with the 1964 Helsinki Declaration and its later amendments.

Open Access This article is licensed under a Creative Commons Attribution 4.0 International License, which permits use, sharing, adaptation, distribution and reproduction in any medium or format, as long as you give appropriate credit to the original author(s) and the source, provide a link to the Creative Commons licence, and indicate if changes were made. The images or other third party material in this article are included in the article's Creative Commons licence, unless indicated otherwise in a credit line to the material. If material is not included in the article's Creative Commons licence and your intended use is not permitted by statutory regulation or exceeds the permitted use, you will need to obtain permission directly from the copyright holder. To view a copy of this licence, visit http://creativecommons.org/licenses/by/4.0/.

\section{References}

Acunzo DJ, Mackenzie G, van Rossum MC (2012) Systematic biases in early ERP and ERF components as a result of high-pass filtering. J Neurosci Methods 209(1):212-218. https://doi.org/10.1016/j. jneumeth.2012.06.011

Agostinho CMS, Scherens A, Richter H, Schaub C, Rolke R, Treede RD, Maier C (2009) Habituation and short-term repeatability of thermal testing in healthy human subjects and patients with chronic non-neuropathic pain. Eur J Pain 13(8):779-785. https:// doi.org/10.1016/j.ejpain.2008.10.002

Akaike H (1974) A new look at the statistical model identification. IEEE Trans Autom Control 19(6):716-723. https://doi. org/10.1109/TAC.1974.1100705

Apkarian AV, Bushnell MC, Treede R-D, Zubieta J-K (2005) Human brain mechanisms of pain perception and regulation in health and disease. Eur J Pain 9(4):463-463. https://doi.org/10.1016/j. ejpain.2004.11.001

Bates D, Mächler M, Bolker B, Walker S (2015) Fitting linear mixed-effects models using lme4. J Stat Softw. https://doi. org/10.18637/jss.v067.i01

Christmann C, Koeppe C, Braus DF, Ruf M, Flor H (2007) A simultaneous EEG-fMRI study of painful electric stimulation. NeuroImage 34(4):1428-1437. https://doi.org/10.1016/j.neuroimage .2006 .11 .006

de Tommaso M, Federici A, Santostasi R, Calabrese R, Vecchio E, Lapadula $\mathrm{G}$ et al (2011) Laser-evoked potentials habituation in fibromyalgia. J Pain 12(1):116-124. https://doi.org/10.1016/j. jpain.2010.06.004

Delorme A, Sejnowski T, Makeig S (2007) Enhanced detection of artifacts in EEG data using higher-order statistics and 
independent component analysis. NeuroImage 34(4):14431449. https://doi.org/10.1016/j.neuroimage.2006.11.004

Doll RJ, Buitenweg JR, Meijer HGE, Veltink PH (2014) Tracking of nociceptive thresholds using adaptive psychophysical methods. Behav Res Methods 46(1):55-66. https://doi.org/10.3758/s1342 8-013-0368-4

Doll RJ, Veltink PH, Buitenweg JR (2015) Observation of timedependent psychophysical functions and accounting for threshold drifts. Atten Percept Psychophys 77(4):1440-1447. https:// doi.org/10.3758/s13414-015-0865-x

Doll RJ, Maten ACA, Spaan SPG, Veltink PH, Buitenweg JR (2016a) Effect of temporal stimulus properties on the nociceptive detection probability using intra-epidermal electrical stimulation. Exp Brain Res 234(1):219-227. https://doi.org/10.1007/s0022 1-015-4451-1

Doll RJ, van Amerongen G, Hay JL, Groeneveld GJ, Veltink PH, Buitenweg JR (2016b) Responsiveness of electrical nociceptive detection thresholds to capsaicin ( $8 \%$ )-induced changes in nociceptive processing. Exp Brain Res 234(9):2505-2514. https://doi. org/10.1007/s00221-016-4655-Z

Faraggi D, Izikson P, Reiser B (2003) Confidence intervals for the 50 per cent response dose. Stat Med 22(12):1977-1988. https://doi. org/10.1002/sim.1368

Garcia-Larrea L, Frot M, Valeriani M (2003) Brain generators of laser-evoked potentials: from dipoles to functional significance. Neurophysiol Clin 33(6):279-292. https://doi.org/10.1016/j.neucl i. 2003.10 .008

Iannetti GD, Hughes NP, Lee MC, Mouraux A (2008) Determinants of laser-evoked EEG responses: pain perception or stimulus saliency? J Neurophysiol 100(2):815-828. https://doi.org/10.1152/ jn.00097.2008

Inui K, Kakigi R (2012) Pain perception in humans: use of intraepidermal electrical stimulation. J Neurol Neurosurg Psychiatry 83(5):551-556. https://doi.org/10.1136/jnnp-2011-301484

Inui K, Tran TD, Hoshiyama M, Kakigi R (2002) Preferential stimulation of A $\delta$ fibers by intra-epidermal needle electrode in humans. Pain 96(3):247-252. https://doi.org/10.1016/S0304 $-3959(01) 00453-5$

Kiernan MC, Mogyoros I, Burke D (1996) Differences in the recovery of excitability in sensory and motor axons of human median nerve. Brain 119(Pt 4):1099-1105. https://doi.org/10.1093/brain /119.4.1099

Lee MC, Mouraux A, Iannetti GD (2009) Characterizing the cortical activity through which pain emerges from nociception. J Neurosci 29(24):7909-7916. https://doi.org/10.1523/JNEUR OSCI.0014-09.2009

Legrain V, Iannetti GD, Plaghki L, Mouraux A (2011) The pain matrix reloaded: a salience detection system for the body. Prog Neurobiol 93(1):111-124. https://doi.org/10.1016/j.pneurobio.2010.10.005

Lehmann D, Skrandies W (1980) Reference-free identification of components of checkerboard-evoked multichannel potential fields. Electroencephalogr Clin Neurophysiol 48(6):609-621. https:// doi.org/10.1016/0013-4694(80)90419-8

Liang M, Lee MC, O’Neill J, Dickenson AH, Iannetti GD (2016) Brain potentials evoked by intraepidermal electrical stimuli reflect the central sensitization of nociceptive pathways. J Neurophysiol 116(2):286-295. https://doi.org/10.1152/jn.00013.2016

Manresa JB, Andersen OK, Mouraux A, van den Broeke EN (2018) High frequency electrical stimulation induces a long-lasting enhancement of event-related potentials but does not change the perception elicited by intra-epidermal electrical stimuli delivered to the area of increased mechanical pinprick sensitivity. PLoS ONE, 13(9). Available at https://www.scopus.com/inwar d/record.uri?eid=2-s2.0-85053139379\&doi=10.1371\%2fjournal. pone. $0203365 \&$ partnerID $=40 \& \mathrm{md} 5=$ efbb2ee $6 \mathrm{f} 95 \mathrm{f} 966 \mathrm{~b} 609 \mathrm{~b}$ 005b615c7ce6. https://doi.org/10.1371/journal.pone.0203365
May A, Rodriguez-Raecke R, Schulte A, Ihle K, Breimhorst M, Birklein F, Jürgens TP (2012) Within-session sensitization and between-session habituation: a robust physiological response to repetitive painful heat stimulation. Eur J Pain 16(3):401-409. https://doi.org/10.1002/j.1532-2149.2011.00023.x

Miyazaki M, Shibasaki H, Kanda M, Xu X, Shindo K, Honda M et al (1994) Generator mechanism of pain-related evoked potentials following $\mathrm{CO}_{2}$ laser stimulation of the hand: scalp topography and effect of predictive warning signal. J Clin Neurophysiol 11(2):242-254. https://doi.org/10.1097/00004691-19940 $3000-00010$

Moscatelli A, Mezzetti M, Lacquaniti F (2012) Modeling psychophysical data at the population-level: the generalized linear mixed model. J Vis 12(11):26-26. https://doi.org/10.1167/12.11.26

Mouraux A, Iannetti GD, Plaghki L (2010) Low intensity intra-epidermal electrical stimulation can activate $\mathrm{A} \delta$-nociceptors selectively. Pain 150(1):199-207. https://doi.org/10.1016/j.pain.2010.04.026

Mouraux A, Marot E, Legrain V (2014) Short trains of intra-epidermal electrical stimulation to elicit reliable behavioral and electrophysiological responses to the selective activation of nociceptors in humans. Neurosci Lett 561(Supplement C):69-73. https://doi. org/10.1016/j.neulet.2013.12.017

Oostenveld R, Praamstra P (2001) The five percent electrode system for high-resolution EEG and ERP measurements. Clin Neurophysiol 112(4):713-719. https://doi.org/10.1016/s1388-2457(00)00527-7

Oostenveld R, Fries P, Maris E, Schoffelen JM (2011) FieldTrip: open source software for advanced analysis of MEG, EEG, and invasive electrophysiological data. Comput Intell Neurosci. https:// doi.org/10.1155/2011/156869

Poulsen AH, Tigerholm J, Meijs S, Andersen OK, Mørch CD (2020) Comparison of existing electrode designs for preferential activation of cutaneous nociceptors. J Neural Eng. https://doi. org/10.1088/1741-2552/ab85b1

Rodriguez-Raecke R, Ihle K, Ritter C, Muhtz C, Otte C, May A (2014) Neuronal differences between chronic low back pain and depression regarding long-term habituation to pain. Eur J Pain (UK) 18(5):701-711. https://doi.org/10.1002/j.1532-2149.2013.00407.x

Schwarz G (1978) Estimating the dimension of a model. Ann Stat 6(2):461-464. Available at www.jstor.org/stable/2958889.

Steenbergen P, Buitenweg JR, Trojan J, van der Heide EM, van den Heuvel T, Flor H, Veltink PH (2012) A system for inducing concurrent tactile and nociceptive sensations at the same site using electrocutaneous stimulation. Behav Res Methods 44(4):924-933. https://doi.org/10.3758/s13428-012-0216-y

Tanner D, Morgan-Short K, Luck SJ (2015) How inappropriate highpass filters can produce artifactual effects and incorrect conclusions in ERP studies of language and cognition. Psychophysiology 52(8):997-1009. https://doi.org/10.1111/psyp.12437

Treede RD, Kief S, Hölzer T, Bromm B (1988) Late somatosensory evoked cerebral potentials in response to cutaneous heat stimuli. Electroencephalogr Clin Neurophysiol 70(5):429-441. https://doi. org/10.1016/0013-4694(88)90020-X

Valeriani M, de Tommaso M, Restuccia D, Le Pera D, Guido M, Iannetti GD et al (2003) Reduced habituation to experimental pain in migraine patients: a $\mathrm{CO}_{2}$ laser evoked potential study. Pain 105(1):57-64. https://doi.org/10.1016/S0304-3959(03)00137-4

van den Berg B, Doll RJ, Mentink ALH, Siebenga PS, Groeneveld GJ, Buitenweg JR (2020) Simultaneous tracking of psychophysical detection thresholds and evoked potentials to study nociceptive processing. Behav Res Methods. https://doi.org/10.3758/s1342 8-019-01338-7

Van den Berg B, Buitenweg JR (2018) Analysis of nociceptive evoked potentials during multi-stimulus experiments using linear mixed models. Paper presented at the 40th Annual International Conference of the IEEE Engineering in Medicine and Biology Society (EMBC). IEEE, Honolulu 
Van der Lubbe RHJ, Blom JHG, De Kleine E, Bohlmeijer ET (2017) Comparing the effects of sustained and transient spatial attention on the orienting towards and the processing of electrical nociceptive stimuli. Int J Psychophysiol 112:9-21. https://doi. org/10.1016/j.ijpsycho.2016.11.015

Vossen CJ, Vossen HG, Joosten EA, Van Os J, Lousberg R (2015) Does habituation differ in chronic low back pain subjects compared to pain-free controls? A cross-sectional pain rating ERP study reanalyzed with the ERFIA multilevel method. Medicine (US). https://doi.org/10.1097/MD.0000000000000865
Wilkinson GN, Rogers CE (1973) Symbolic description of factorial models for analysis of variance. J Royal Stat Soc Series C (Appl Stat) 22(3):392-399. https://doi.org/10.2307/2346786

Zucker RS, Regehr WG (2002) Short-term synaptic plasticity. Annu Rev Physiol 64:355-405. https://doi.org/10.1146/annurev.physi ol.64.092501.114547

Publisher's Note Springer Nature remains neutral with regard to jurisdictional claims in published maps and institutional affiliations. 